\title{
Mycobacterium genus and tRNA arrays
}

\author{
Sergio Mascarenhas Morgado/ ${ }^{+}$, Ana Carolina Paulo Vicente \\ Fundação Oswaldo Cruz-Fiocruz, Instituto Oswaldo Cruz, Laboratório de Genética Molecular de Microrganismos, Rio de Janeiro, RJ, Brasil
}

The presence of tRNA array, a region with high tRNA gene number and density, has been demonstrated in Mycobacterium genus. However, a recent phylogenomic study revealed the existence of five distinct monophyletic groups (genera) within this genus. Considering this new scenario, and based on in-silico analyses, we have identified and characterised the abundance and diversity of tRNA array units within Mycobacterium, Mycolicibacterium gen. nov., Mycolicibacillus gen. nov., and Mycobacteroides gen. nov. The occurrence and prevalence of tRNA arrays among the genera belonging to Actinobacteria indicate their possible role in the organismal fitness.

Key words: tRNA array - Mycobacterium - Mycolicibacterium - Mycolicibacillus - Mycobacteroides - Actinobacteria

The tRNA genes are generally distributed along the genomes and are eventually arranged in small clusters (up to five genes), as seen in mitogenomes. ${ }^{(1,2)}$ In contrast to these small clusters, genomic regions with a high number (from 19 to 39 tRNA genes) and density of tRNA genes have been identified and characterised in archaea, eukaryotes, bacteria, and viruses..$^{(3,4,5,6,7,8,9,10)}$ As far as the bacteria are concerned, it was initially thought that such structures would occur only in a few phyla, particularly Firmicutes. ${ }^{(8)}$ However, tRNA arrays have been revealed to be abundant among Mycobacterium; they have been found in chromosomes and/or plasmids of several species, including fast- and slow-growing species. ${ }^{(9)}$ Recently, a study proposed a reclassification for the Mycobacterium genus into five clades; one of them has been associated with the older Mycobacterium genus and four new clades have been suggested: Mycolicibacterium gen. nov., Mycolicibacter gen. nov., Mycolicibacillus gen. nov., and Mycobacteroides gen. nov. ${ }^{(11)}$ In the face of this reclassification and due to the importance of this phylum, which encompasses organisms of agricultural, biotechnological, clinical, and ecological importance, we questioned if tRNA arrays occurred in all five clades (genera), particularly in Mycobacterium. In order to verify the presence of tRNA arrays in these five proposed clades (genera), we screened the representative genomes belonging to them. The tRNA arrays were identified and characterised based on a previous methodology for $M y$ cobacterium genus. ${ }^{(9)}$ A phylogenetic tree, based on the core genome of these bacteria, was build (cgMLSA) considering the representative genomes harbouring tRNA arrays, from each of the proposed clade (36 genomes in total). The cgMLSA analysis was performed by GET HOMOLOGUES v3.05 ${ }^{(12)}$ using parameters of minimum coverage of $\geq 70 \%$ and identity of $\geq 40 \%$. The sequences were aligned using MAFFT v7.271 ${ }^{(13)}$ and trimmed using trimAL v1.2.(14) A neighbour joining tree, based on

doi: 10.1590/0074-02760180443

Financial support: This study was financed in part by the CAPES

(Finance Code 001).

+ Corresponding author: sergio.morgado@ioc.fiocruz.br

(D) https://orcid.org/0000-0003-4877-7639

Received 17 September 2018

Accepted 28 March 2019
806 concatenated genes $(\sim 831 \mathrm{~kb})$, was constructed using Seaview v4.7 $7^{(15)}$ with 1000 bootstrap replicates and edited using iTOL. ${ }^{(16)}$ This analysis revealed that the tRNA arrays occur in species from Mycobacterium genus as well as in all new proposed genera, except $\mathrm{Mycol}$ icibacter gen. nov. (Figure). In fact, there is a bias concerning this new genus due to low number of genomes/ drafts available in NCBI; therefore, the occurrence of the tRNA arrays cannot be completely ruled out. The arrays presented a tRNA repertoire that varied from 16 to 20 isotypes, most of them presenting the 20 universal tRNA isotypes. Despite the large tRNA repertoire provided by the arrays, these tRNA isotypes are redundant compared to those of non-arrayed tRNAs. However, few genomes (genome labels highlighted in bold in Figure) had an increment in the number of isoacceptor species (one or two), being the isoacceptor tRNA-Thr ${ }^{\text {AGU }}$ the one present in all these genomes, except in $M$. koreense KCTC 19819, that presented tRNA-His ${ }^{\mathrm{AUG}}$ isoacceptor. Most of the arrayed tRNAs seem to be functional as only a few genes (one or two per array in 11/21 genomes harbouring tRNA array) were annotated as pseudogenes (isotype letters highlighted in bold in Figure). Interestingly, similar tRNA isotype organisation of some tRNA arrays was identified in genomes from Mycobacterium species and from the newly proposed genera. In a previous study, based on the tRNA gene isotype organisation, the tRNA arrays from Mycobacterium genomes were characterised in three groups and a singleton, ${ }^{(9)}$ as also has been observed in this study. The tRNA array group 2 (represented by squares in the Figure) is present in the genomes from Mycolicibacterium gen. nov. and Mycobacteroides gen. nov., while the tRNA array group 1 (represented by circles in the Figure) and the tRNA array singleton (represented by triangles in the Figure) are exclusively observed in the genomes from Mycobacteroides gen. nov. and Mycolicibacillus gen. nov., respectively. Taken together, these results show the presence of tRNA arrays in the Mycobacterium genus even after the its reclassification as well as in other Actinobacteria genera; this is in contrast with the previous study that revealed lower prevalence of such structures in this phylum. ${ }^{(8)}$ Moreover, the dispersion of tRNA arrays with the same isotype organisation among different species and genera corroborates the hypothesis of their association 


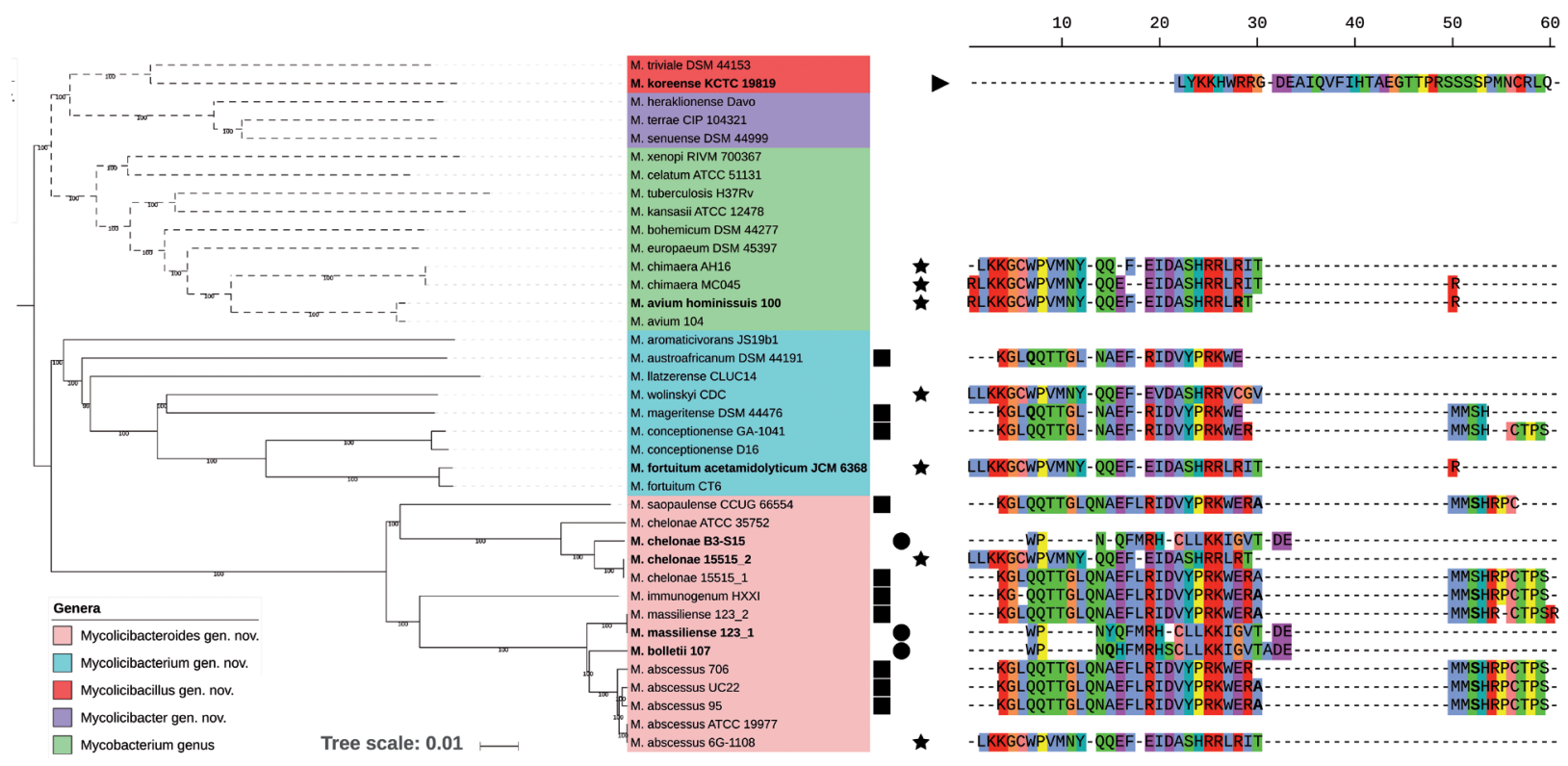

Phylogenetic tree of Mycobacterium, Mycolicibacterium gen. nov., Mycolicibacter gen. nov., Mycolicibacillus gen. nov., and Mycobacteroides gen. nov. (left side). The genera are discriminated by the background colour and the dotted branches correspond to the slow-growing species. The genomes highlighted in bold represent those with an increase in the isoacceptor species. The symbols indicate tRNA array group: circle, group 1; square, group 2; star, group 3; triangle, singleton. The tRNA isotype organisation of each array is represented using the single-letter amino acid code (right side). The isotypes in bold correspond to the genes annotated as pseudogenes.

with mobile elements. Indeed, tRNA arrays were associated with horizontal gene transfer events mediated by the plasmids and bacteriophages. ${ }^{(8,9)}$ Particularly, tRNA gene clusters have been shown to be prevalent among mycobacteriophages. ${ }^{(5,17)}$ Even though the role of these elements is still debated, ${ }^{(3,6,18)}$ their occurrence and prevalence among eukaryotes, prokaryotes, and viruses indicate a possible positive implication in the organismal fitness.

\section{AUTHORS' CONTRIBUTION}

ACPV conceived and supervised all the steps involved in this study and SMM carried out the in silico analysis. Both, ACPV and SMM, discussed the results and wrote the manuscript.

\section{REFERENCES}

1. Friedrich A, Jung PP, Hou J, Neuvéglise C, Schacherer J. Comparative mitochondrial genomics within and among yeast species of the Lachancea genus. PLoS One. 2012; 7(10): e47834.

2. Li E, Li X, Wu X, Feng G, Zhang M, Shi H, et al. Complete nucleotide sequence and gene rearrangement of the mitochondrial genome of Occidozyga martensii. J Genet. 2014; 93(3): 631-41.

3. Bailly-Bechet M, Vergassola M, Rocha E. Causes for the intriguing presence of tRNAs in phages. Genome Res. 2007; 17(10): 1486-95.

4. Bermudez-Santana C, Attolini CS, Kirsten T, Engelhardt J, Prohaska SJ, Steigele S, et al. Genomic organization of eukaryotic tRNAs. BMC Genomics. 2010; 11: 270.

5. Pope WH, Anders KR, Baird M, Bowman CA, Boyle MM, Broussard GW, et al. Cluster M mycobacteriophages Bongo, PegLeg, and Rey with unusually large repertoires of tRNA isotypes. J Virol. 2014; 88(5): 2461-80.

6. Puerto-Galán L, Vioque A. Expression and processing of an unusual tRNA gene cluster in the cyanobacterium Anabaena sp. PCC 7120. FEMS Microbiol Lett. 2012; 337(1): 10-7.

7. Senčilo A, Jacobs-Sera D, Russell DA, Ko CC, Bowman CA, Atanasova NS, et al. Snapshot of haloarchaeal tailed virus genomes. RNA Biol. 2013; 10(5): 803-16.
8. Tran TTT, Belahbib H, Bonnefoy V, Talla E. A comprehensive tRNA genomic survey unravels the evolutionary history of tRNA arrays in prokaryotes. Gen Biol Evol. 2016; 8(1): 282-95.

9. Morgado SM, Vicente ACP. Beyond the limits: tRNA array units in Mycobacterium genomes. Front Microbiol. 2018; 9: 1042.

10. Morgado SM, Vicente ACP. Exploring tRNA gene cluster in archaea. Mem Inst Oswaldo Cruz. 2019; 114: e180348.

11. Gupta RS, Lo B, Son J. Phylogenomics and comparative genomic studies robustly support division of the genus Mycobacterium into an emended genus Mycobacterium and four novel genera. Front Microbiol. 2018; 9: 67.

12. Contreras-Moreira B, Vinuesa P. GET_HOMOLOGUES, a versatile software package for scalable and robust microbial pangenome analysis. Appl Environ Microbiol. 2013; 79(24): 7696-7701.

13. Katoh K, Standley DM. MAFFT multiple sequence alignment software version 7: improvements in performance and usability. Mol Biol Evol. 2013; 30(4): 772-80.

14. Capella-Gutiérrez S, Silla-Martínez JM, Gabaldón T. trimAl: a tool for automated alignment trimming in large-scale phylogenetic analyses. Bioinformatics. 2009; 25(15): 1972-3.

15. Gouy M, Guindon S, Gascuel O. SeaView version 4: a multiplatform graphical user interface for sequence alignment and phylogenetic tree building. Mol Biol Evol. 2010; 27(2): 221-4.

16. Letunic I, Bork P. Interactive tree of life (iTOL) v3: an online tool for the display and annotation of phylogenetic and other trees. Nucleic Acids Res. 2016; 44: W242-5.

17. Delesalle VA, Tanke NT, Vill AC, Krukonis GP. Testing hypotheses for the presence of tRNA genes in mycobacteriophage genomes. Bacteriophage. 2016; 6: e1219441.

18. Alamos P, Tello M, Bustamante P, Gutiérrez F, Shmaryahu A, Maldonado J, et al. Functionality of tRNAs encoded in a mobile genetic element from an acidophilic bacterium. RNA Biol. 2017; 15(4-5): 518-527. 